\title{
Dynamic Modeling and Control of Power-Split HEV with Multi-phase Electric Machines under Fault Condition
}

\author{
Federica Grossi ${ }^{1}$, Marco $\mathrm{Fei}^{1}$ and Roberto Zanasi ${ }^{1}$
}

\begin{abstract}
The aim of this paper is to provide a dynamic model of the Toyota Hybrid System (THS) for simulation and control purposes in the case of fault of one of the electric machines. The two three-phase electric machines that are commonly used in THS are here replaced by two five-phase machines. The model of the whole system is realized by using the Power-Oriented Graphs modeling technique and includes the dynamics of the engine, electric machines, planetary gear, transmission and vehicle. A rule-based control strategy is used to operate the vehicle in different operation modes and a faulttolerant control is applied in the case of electrical machine failure. Simulation results are given in both healthy and fault condition to show the effectiveness of the dynamic model and the robustness of the proposed control.
\end{abstract}

\section{INTRODUCTION}

The development of new control strategies and/or new architectures for hybrid electric vehicles (HEVs) requires a deep knowledge of the dynamic behavior and the dynamic interaction among all subsystems constituting HEVs [1]. Therefore it is essential to have precise and reliable simulative models to reduce the effort and the cost in the testing phase [2], [3]. The interest in hybrid vehicle simulation has grown in the past years and led to the development of many computer programs to describe the operation of hybrid power trains. Some examples of steady-state and quasi-steady-state simulators are: JANUS [4], ADVISOR [5], PSAT [6] and Autonomie [7] from Argonne National Laboratory, CarSim from Mechanical Simulation Corporation and many others. Although steady-state or quasi-steady-state models allow to achieve fast computation time, they do not permit to capture the transient behavior and may be unsuitable for systems in which this is a crucial aspect. Therefore dynamic models can be used to make accurate dynamic analysis and simulation of the system and to design effective control strategies.

Nowadays, a lot of modeling methods are available to describe complex dynamic systems such as Bond Graphs [8]-[9], Causal Ordering Graph [10], Power-Oriented Graphs [11]-[12], Energetic Macroscopic Representation [13]-[15]. All these techniques are energy-based techniques which use power interaction between subsystems as the basic concept for modeling and clearly show how the power flows within the system. In this paper the Power-Oriented Graphs (POG) modeling technique is exploited to build the dynamic model of the power-split Toyota Hybrid System (THS) [16]-[18]. The basic features of POG are stated in Appendix. Taking

\footnotetext{
${ }^{1}$ Federica Grossi, Marco Fei and Roberto Zanasi are with Engineering Department, University of Modena e Reggio Emilia, Modena, Italy \{federica.grossi, marco.fei, roberto.zanasi\}@unimore.it.
}

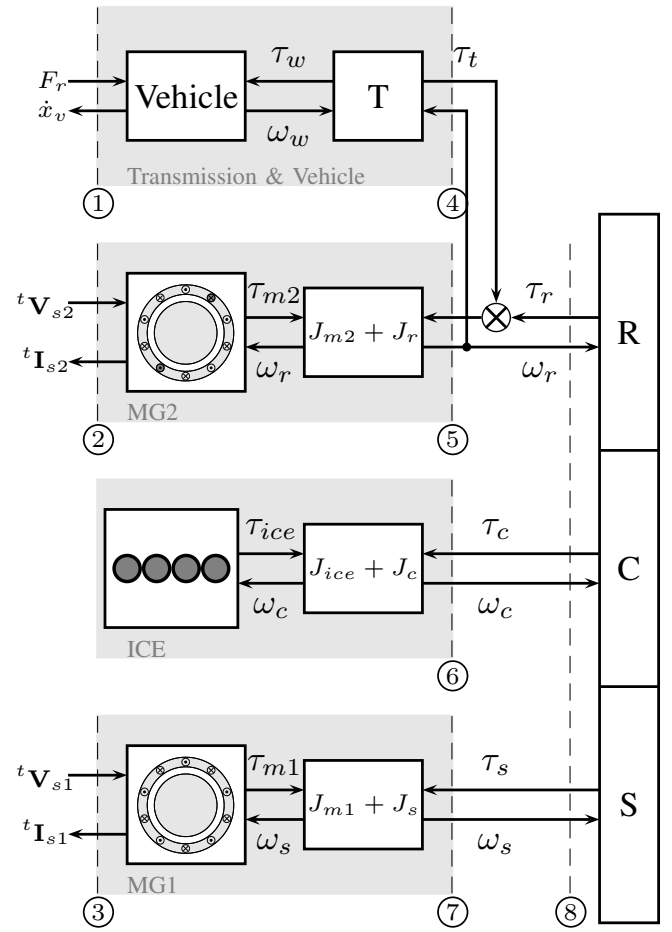

Fig. 1. Scheme of the considered power structure of the vehicle.

advantage from the POG modular structure, the model of a complex physical system can be obtained by interconnecting the models of the subsystems that interact each other through their power ports. Following this approach the whole THS has been obtained modeling the main subsystems. In this model two 5-phase synchronous machines are used because multi-phase electrical motors offer many advantages compared to three-phase machines, see [19] and [20]. The model is then implemented in Simulink and used to simulate the vehicle when one electric machine is in open-phase fault condition. The control of the vehicle powertrain is designed starting from the analysis of the power flows within the system. Different operation modes are possible and they will be examined in the paper.

\section{POG MODELING OF THE TOYOTA HYBRID SYSTEM}

The power-split configuration considered in the paper is that of the Toyota Hybrid System (THS-II) of the Prius [16]-[18]: it includes an internal combustion engine (ICE), two multi-phase Permanent Magnet Synchronous Machines (MG1 and MG2), a transmission and the vehicle. The powersplit is provided by a planetary gear: the ICE is rigidly 


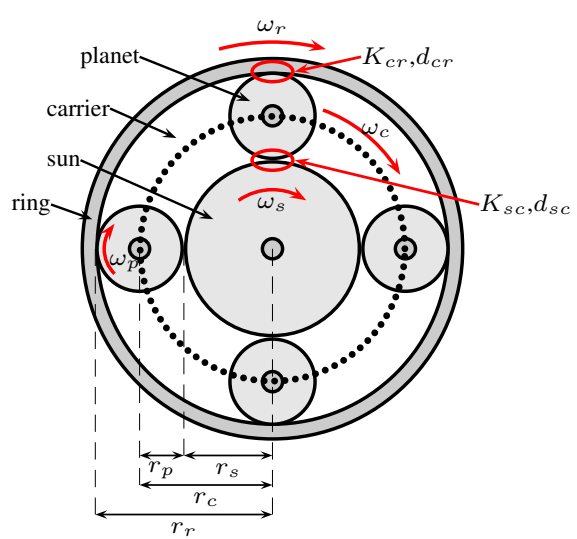

Fig. 2. Planetary gear and related parameters.

connected to the Carrier (C), MG1 is connected to the Sun (S), MG2 and the vehicle are connected to the Ring (R). In Fig. 1 the scheme of such system is shown: the dashed lines denoted by numbers indicate the power sections within the system. These are the physical power sections through which the power flows among the subsystems. The power sections shown in Fig. 1 correspond to the power sections indicated by the same numbers in the POG schemes of the considered subsystems presented in the next part of the paper. In this paper the devices for storing electrical energy, such as batteries, supercapacitors and others, are not taken into account.

\section{A. Planetary Gear modeling}

The considered planetary gear is shown in Fig. 2. The main parameters of the system are: $r_{s}, r_{c}, r_{r}$ and $r_{p}$ are the sun, carrier, ring and planet radii; $J_{s}, b_{s}, J_{c}, b_{c}, J_{r}, b_{r}, J_{p}$ and $b_{p}$ are the inertia and linear friction coefficients of the sun, carrier, ring and planet, respectively; $K_{s c}, d_{s c}, K_{c r}$ and $d_{c r}$ are the stiffness and friction coefficients of the sun-carrier and carrier-ring elastic elements, respectively.

The planetary gear inertias of the sun, carrier and ring are rigidly connected to the inertias of the MG1, ICE and MG2, respectively, therefore a reduced elastic model of the planetary gear is used, see [21] where the POG technique is exploited to obtain different reduced models of the planetary gear using proper mathematical transformations. As shown in Fig. 1 the equivalent inertias are considered: $J_{m 2}+J_{r}$ for MG2 and ring, $J_{i c e}+J_{c}$ for ICE and carrier and $J_{m 1}+J_{s}$ for MG1 and sun. The dynamic equations of the elastic model of the planetary gear in state space form (1), see [22], are given in Fig. 3. There is a direct correspondence between the POG schemes and the state space dynamic equations, therefore equations in (1) can be represented by the POG scheme shown in Fig 4. Note that this elastic model has the speeds as input and the torques as output. The following kinematics Willis relation [23] between angular velocities as function of the gear radii:

$$
r_{s} \omega_{s}+r_{r} \omega_{r}=\left(r_{s}+r_{r}\right) \omega_{c}=2 r_{c} \omega_{c}
$$

can be obtained from equation (1) in steady-state condition, i.e. $\dot{\mathbf{x}}=0$.

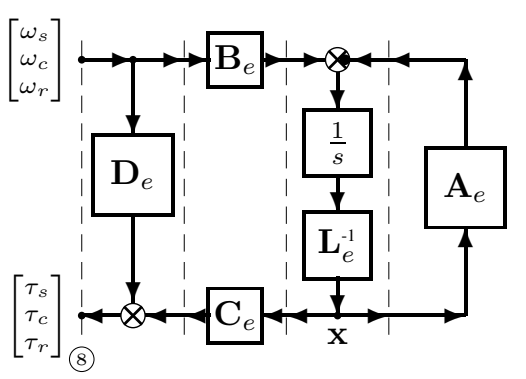

Fig. 4. POG block scheme of the elastic model of the planetary gear.

\section{B. Internal Combustion Engine model}

A POG simplified model of the engine that considers only the main mechanical dynamics is proposed in Fig. 5: $J_{i c e}+J_{c}$ is the inertia of the engine shaft and carrier, $b_{i c e}$ is the friction coefficient, $\tau_{i c e}$ is the torque provided by the engine and $\tau_{i c e, d}$ is the requested torque. The engine torque-speed map provides the maximum engine torque $\tau_{i c e, \max }$ for a given engine speed $\omega_{c}$. The provided torque is defined as $\tau_{i c e}=$ $\min \left\{\tau_{\text {ice,d }}, \tau_{\text {ice, } \max }\right\}$.

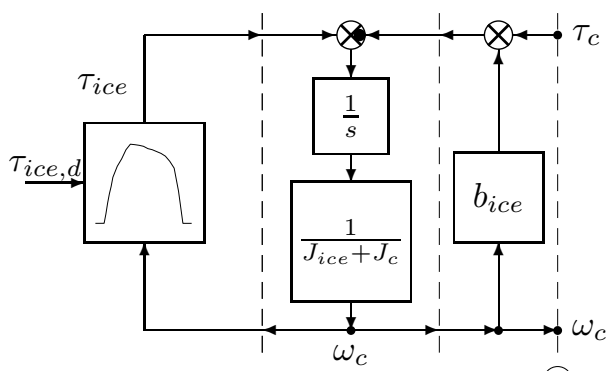

Fig. 5. POG scheme of the ICE model.

\section{Electrical motors modeling}

The fault tolerant capability of multi-phase motor drives and the possibility to enhance the motor torque by injecting higher order stator current harmonics, see [19] and [20], make the multi-phase synchronous machines suitable for Electric and Hybrid Electric Vehicles where reliability and power density are very important issues.

The basic structure of a permanent magnet synchronous motor with five concentrated winding in star connection is shown in Fig. 6. Under the assumptions of regularity of the design, no reluctance effect and no iron saturation, the dynamic model of the 5-phase motor can be expressed by the following equation system:

$$
\left[\begin{array}{c|c}
{ }^{t} \mathbf{L}_{s} & \mathbf{0} \\
\hline \mathbf{0} & J_{m}
\end{array}\right]\left[\begin{array}{c}
{ }^{t} \dot{\mathbf{I}}_{s} \\
\hline \dot{\omega}_{m}
\end{array}\right]=-\left[\begin{array}{c|c}
{ }^{t} \mathbf{R}_{s} & { }^{t} \mathbf{K}_{\tau}(\theta) \\
\hline-{ }^{t} \mathbf{K}_{\tau}^{\mathrm{T}}(\theta) & b_{m}
\end{array}\right]\left[\frac{{ }^{t} \mathbf{I}_{s}}{\omega_{m}}\right]+\left[\frac{{ }^{t} \mathbf{V}_{s}}{-\tau_{e}}\right]
$$

where the stator current and voltage vectors are:

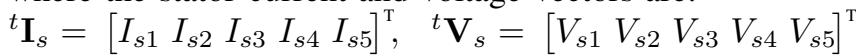
and the $i$-th phase voltage is referred to the common voltage $V_{s 0}$ as: $V_{s i}=V_{i}-V_{s 0}$.

The input $\tau_{e}$ is the external load torque acting on the rotor. The resistance matrix is diagonal: ${ }^{t} \mathbf{R}_{s}=R_{s} \mathbf{I}_{5}$, where $R_{s}$ is the stator phase resistance and $\mathbf{I}_{5}$ is the 5 -dimension identity 


$$
\begin{aligned}
& \underbrace{\left[\begin{array}{ccc}
\frac{1}{K_{s c}} & 0 & 0 \\
0 & J_{p} & 0 \\
0 & 0 & \frac{1}{K_{c r}}
\end{array}\right]}_{\mathbf{L}_{e}} \underbrace{\left[\begin{array}{c}
\dot{F}_{s c} \\
\dot{\omega}_{p} \\
\dot{F}_{c r}
\end{array}\right]}_{\dot{\mathbf{x}}}=\underbrace{\left[\begin{array}{ccc}
0 & r_{p} & 0 \\
-r_{p} & -r_{p}^{2} d_{s c}-b_{p}-r_{p}^{2} d_{c r} & -r_{p} \\
0 & r_{p} & 0
\end{array}\right]}_{-\mathbf{A}_{e}} \underbrace{\left[\begin{array}{c}
F_{s c} \\
\omega_{p} \\
F_{c r}
\end{array}\right]}_{\mathbf{x}}+\underbrace{\left[\begin{array}{ccc}
r_{s} & -r_{s} & 0 \\
-r_{s} d_{s c} r_{p} & r_{s} d_{s c} r_{p}-r_{r} d_{c r} r_{p} & r_{r} d_{c r} r_{p} \\
0 & r_{r} & -r_{r}
\end{array}\right]}_{\mathbf{B}_{e}} \underbrace{\left[\begin{array}{c}
\omega_{s} \\
\omega_{c} \\
\omega_{r}
\end{array}\right]}_{\mathbf{u}}
\end{aligned}
$$

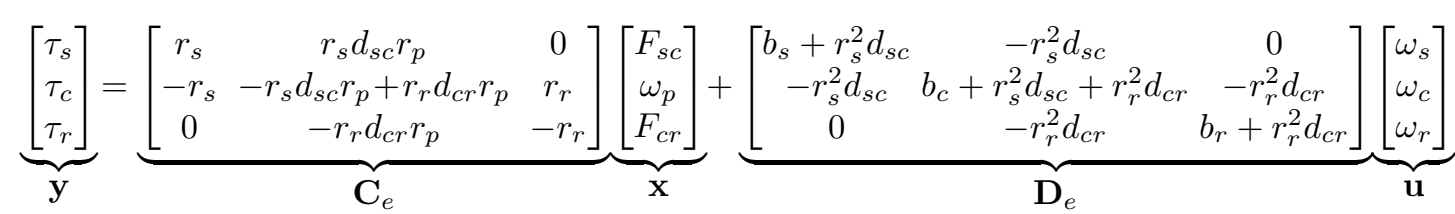

Fig. 3. State space equations of the planetary gear elastic model.

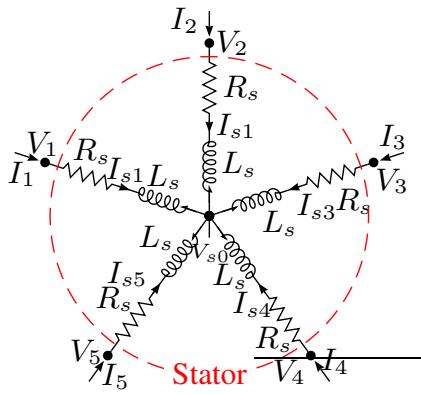

Fig. 6. Basic structure of a star-connected five-phase synchronous motor.

matrix. The symmetric inductance matrix ${ }^{t} \mathbf{L}_{s}$ is:

$$
{ }^{t} \mathbf{L}_{s}=\left[\begin{array}{ccccc}
L_{s} & M_{s} \cos \left(\gamma_{s}\right) & M_{s} \cos \left(2 \gamma_{s}\right) & M_{s} \cos \left(3 \gamma_{s}\right) & M_{s} \cos \left(4 \gamma_{s}\right) \\
M_{s} \cos \left(\gamma_{s}\right) & L_{s} & M_{s} \cos \left(\gamma_{s}\right) & M_{s} \cos \left(2 \gamma_{s}\right) & M_{s} \cos \left(3 \gamma_{s}\right) \\
M_{s} \cos \left(2 \gamma_{s}\right) & M_{s} \cos \left(\gamma_{s}\right) & L_{s} & M_{s} \cos \left(\gamma_{s}\right) & M_{s} \cos \left(2 \gamma_{s}\right) \\
M_{s} \cos \left(3 \gamma_{s}\right) & M_{s} \cos \left(2 \gamma_{s}\right) & M_{s} \cos \left(\gamma_{s}\right) & L_{s} & M_{s} \cos \left(\gamma_{s}\right) \\
M_{s} \cos \left(4 \gamma_{s}\right) & M_{s} \cos \left(3 \gamma_{s}\right) & M_{s} \cos \left(2 \gamma_{s}\right) & M_{s} \cos \left(\gamma_{s}\right) & L_{s}
\end{array}\right]
$$

where $L_{s}$ is the self induction coefficient, $M_{s}$ is the maximum value of the stator mutual inductance and $\gamma_{s}$ is the basic angular displacement $\gamma_{s}=2 \pi / 5$.

According to the magnetic co-energy method the torque $\tau_{m}$ and the back-electromotive force ${ }^{t} \mathbf{E}_{s}$ can be expressed as:

$$
\tau_{m}={ }^{t} \mathbf{K}_{\tau}^{\mathrm{T}}{ }^{t} \mathbf{I}_{s}, \quad{ }^{t} \mathbf{E}_{s}={ }^{t} \mathbf{K}_{\tau} \omega_{m}
$$

where the torque vector ${ }^{t} \mathbf{K}_{\tau}(\theta)$ (also known as speed normalized back electromotive force) is:

$$
{ }^{t} \mathbf{K}_{\tau}(\theta)=\left[\begin{array}{lllll}
K_{\tau 1} & K_{\tau 2} & K_{\tau 3} & K_{\tau 4} & K_{\tau 5}
\end{array}\right]^{\mathrm{T}} .
$$

The components $K_{\tau h}$ are function of the electric angle $\theta$ and of the harmonics $a_{n}$ of the rotor flux as follows:

$$
K_{\tau h}=-p \varphi_{c} \sum_{n=1: 2}^{\infty} n a_{n} \sin \left(n\left(\theta-h \gamma_{s}\right)\right), h=1,2, \ldots, 5
$$

where $p$ is the number of polar expansions and $\varphi_{c}$ is the maximum value of the rotor flux chained with the first stator phase. The POG block scheme of the synchronous motor (3) is shown in Fig. 7. In [24] the simulation of an open phase fault has been proposed in rotating reference frame considering that a fusible element opens the phase circuit. However using this method a system time constant becomes very small then the simulation needs a very small

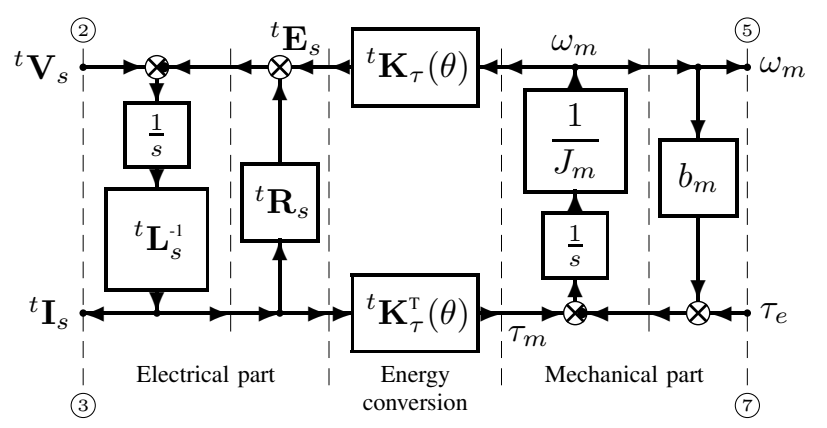

Fig. 7. POG block scheme of the dynamic model of the multi-phase synchronous motors MG1 and MG2 in the fixed reference frame.

integration step thus increasing the simulation time. Since in case of fault, in rotating reference frame, the transformed resistance matrix becomes full (instead of diagonal), in [25] the simulation of an open phase fault is proposed in fixed reference frame using an additional internal voltage such that the steady-state currents of faulty phases are zero. In this way a short simulation time is achieved because the failures are simulated without increasing the phases resistance.

\section{Model of the transmission and vehicle}

The POG model of the transmission and vehicle is shown in Fig. 8: $K_{t}$ is the transmission stiffness, $d_{t}$ is the transmission friction coefficient, $R_{t}$ is the transmission ratio, $J_{w}$ is the wheel inertia, $R_{w}$ is the wheel radius, $K_{w}$ is the tire longitudinal stiffness, $b_{w}$ is the friction coefficient and $M_{v}$ is the mass of the vehicle. The torque $\tau_{t}$ is the transmission torque, $\tau_{w}$ is the wheel torque and $\tau_{b}$ is the braking torque. The resistance force $F_{r}$ represents the forces applied by the environment and can take into account the air resistance, the slope of the road and other external forces.

\section{Control Strategy}

The control algorithms generally used in many HEVs are rule-based [18], [26]. The control strategy for the THS can be designed in two levels. The vehicle control level governs the dynamic behavior of the vehicle, while the components control level actuates the reference signals from the vehicle control level to regulate the electrical motors and engine operation. 


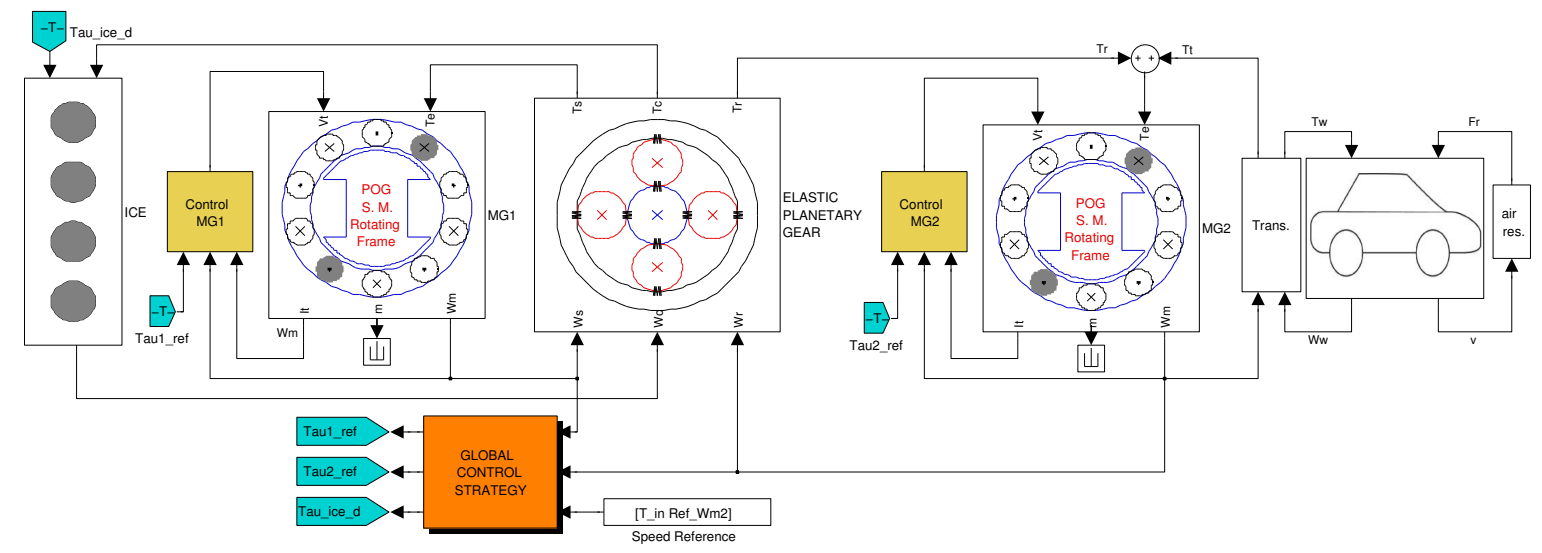

Fig. 9. Simulink block scheme of the power-split HEV.

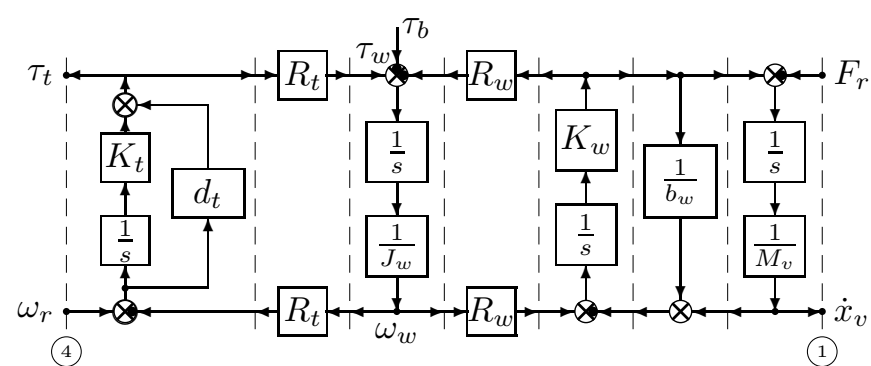

Fig. 8. POG scheme of the model of transmission and vehicle.

\section{Vehicle control level}

Starting from the vehicle reference speed $\dot{x}_{v, d}$ and the estimated power requirement $P_{v e}$, this control generates the torque reference for the electric machines $\tau_{m 1, d}$ and $\tau_{m 2, d}$ and for the engine $\tau_{i c e, d}$. With this control the engine operates in its optimal operating region on the torque-speed plane in which the fuel efficiency is high. The control should manage also the SOC level, but in this work batteries are not modeled or equivalently they are considered always fully charged. The different operation modes and conditions, see [18] and [27], are:

A Driving at low speed: the engine is off and the traction power is all given by MG2.

B Normal driving conditions: the traction power is supplied by the engine operating in its optimal region and by the electric motor MG2, while MG1 operates as generator to supply MG2 (Normal energy flow).

C Boosting: during full throttle acceleration the traction power is given by the engine and by motor MG2.

D High speed cruising: to keep the engine constantly at a low speed, where efficiency is high, MG1 operates as motor and MG2 operates as generator (Energy recirculation flow).

E Deceleration: the engine turns off and MG2 operates as generator recuperating the kinetic energy. When the braking power exceeds a limit value a friction brake is activated.

\section{Components control level}

The reference torques provided by the vehicle control level are the inputs of the motors and engine drives.
Concerning the motor drive, the desired current vector ${ }^{t} \mathbf{I}_{s, d}$, which provides the desired torque $\tau_{m, d}$ minimizing the power dissipation, is the vector with the minimum modulus parallel to the torque vector ${ }^{t} \mathbf{K}_{\tau}(\theta)$ :

$$
{ }^{t} \mathbf{I}_{s, d}(\theta)=\frac{{ }^{t} \mathbf{K}_{\tau}(\theta)}{\left|{ }^{t} \mathbf{K}_{\tau}(\theta)\right|^{2}} \tau_{m, d} .
$$

When an open-phase fault occurs the related phase current does not contribute any more to the torque generation. In this case the open phase constraint can be taken into account projecting the torque vector onto the subspace generated by the healthy currents as follows:

$$
{ }^{t} \mathbf{K}_{\tau}(\theta)=\left[\mathbf{I}_{5}-{ }^{\underline{t}} \mathbf{B}\left({ }^{\underline{t}} \mathbf{B}^{\mathrm{T}}{ }^{\underline{t}} \mathbf{B}\right)^{-1}{ }^{\underline{t}} \mathbf{B}^{\mathrm{T}}\right]{ }^{t} \mathbf{K}_{\tau}(\theta)
$$

where the constraint matrix ${ }^{t} \mathbf{B}=\left[\mathbf{1}^{5} \mathbf{e}_{f}^{5}\right]$ is composed by vector $\mathbf{1}^{5}=\left[\begin{array}{lll}1 & 11 & 1\end{array}\right]^{\mathrm{T}}$ (to impose the star connection constraint) and by the $f$-th standard basis vector $\mathbf{e}_{f}^{5}$ (to select the $f$-th faulty component). The reference current vector in fault condition ${ }^{t_{f}} \mathbf{I}_{s, d}$ is:

$$
{ }^{t_{f}} \mathbf{I}_{s, d}(\theta)=\frac{{ }^{t_{f}} \mathbf{K}_{\tau}(\theta)}{\left|{ }^{t_{f}} \mathbf{K}_{\tau}(\theta)\right|^{2}} \tau_{d} .
$$

All the details of this fault-tolerant control are given in [28] where the current limit for safe operation in fault condition is taken into account.

The engine regulator uses the desired torque $\tau_{i c e, d}$ and delivers the engine torque $\tau_{i c e}$ which cannot exceed the maximum torque $\tau_{i c e, \max }$ for a given engine speed $\tau_{i c e}=$ $\min \left\{\tau_{i c e, d}, \tau_{i c e, \max }\right\}$, see Fig. 5 .

\section{Simulation OF The THS}

The POG schemes presented in this paper have been connected through their power ports like in Fig. 1 and translated into the Simulink model of Fig. 9. Note that the submodels of the ICE, Planetary Gear, electric machines MG1 and MG2, transmission, vehicle and environment forces are masked and contain the POG models presented in the previous sections. The orange block in Fig. 9 exploits the rule-based global control strategy of the vehicle and the yellow blocks 
Table I: Main parameters of the system

\begin{tabular}{|l|c|c|c|}
\hline \multicolumn{4}{|c|}{ Planetary Gear } \\
\hline ring number of teeth & $N_{r}$ & 78 & \\
\hline sun number of teeth & $N_{s}$ & 30 & \\
\hline planet number of teeth & $N_{p}$ & 23 & \\
\hline ring moment of inertia & $J_{r}$ & 0.0081 & $\mathrm{~kg} \mathrm{~m}^{2}$ \\
\hline sun moment of inertia & $J_{s}$ & $1.4810^{-4}$ & $\mathrm{~kg} \mathrm{~m}^{2}$ \\
\hline carrier moment of inertia & $J_{c}$ & 0.0071 & $\mathrm{~kg} \mathrm{~m}^{2}$ \\
\hline \multicolumn{4}{|c|}{ Engine } \\
\hline moment of inertia & $J_{i c e}$ & 0.2596 & $\mathrm{~kg} \mathrm{~m}^{2}$ \\
\hline maximum power & $P_{m a x} @ 5200 \mathrm{rpm}$ & 73 & $\mathrm{~kW}$ \\
\hline \multicolumn{4}{|c|}{ Electric machines } \\
\hline number of phases & $m_{s}$ & 5 & \\
\hline resistance & $R_{s 1}, R_{s 2}$ & $0.5,0.8$ & $\Omega$ \\
\hline self inductance & $L_{s 1}, L_{s 2}$ & 8,11 & $\mathrm{mH}$ \\
\hline mutual inductance & $M_{s 1}, M_{s 2}$ & 3,5 & $\mathrm{mH}$ \\
\hline moment of inertia & $J_{m 1}, J_{m 2}$ & $20.1,28.1$ & $\mathrm{~g} \mathrm{~m}$ \\
\hline \multicolumn{4}{|c|}{ Vehicle and Transmission } \\
\hline vehicle mass & $M_{v}$ & 1600 & $\mathrm{~kg}$ \\
\hline wheel moment of inertia & $J_{w}$ & 1.06 & $\mathrm{~kg} \mathrm{~m}$ \\
\hline wheel radius & $R_{w}$ & 32.55 & $\mathrm{~cm}$ \\
\hline drag coefficient & $\alpha$ & 0.254 & \\
\hline transmission ratio & $R_{t}$ & 4.113 & \\
\hline
\end{tabular}

implement the torque control (also for the fault-tolerant control) of the electric machines. The main parameters of the system are reported in Table I. The simulations for both cases of normal and fault operation consider the Extra Urban Driving Cycle (EUDC).

\section{A. Simulation in normal operation}

The simulated dynamic behavior of the HEV is shown in Fig. 10, 11 and 12. The different operation modes are denoted by letters between vertical dashed lines in Fig. 10 where the horizontal red dashed line indicates the threshold speed for turning on the engine (below $15 \mathrm{~km} / \mathrm{h}$ the engine is off). In mode A the vehicle is in pure electric mode: engine power $P_{i c e}=0$, MG1 power $P_{m 1}=0$ and MG2 power $P_{m 2}>0$, see Fig. 12. In mode $\mathrm{C}$, when the vehicle is accelerating and the speed is greater than $15 \mathrm{~km} / \mathrm{h}$, the ICE starts to help the traction $P_{i c e}>0$. In mode B (normal driving) the traction power is supplied by both the engine and MG2 (i.e. $P_{\text {ice }}>0$ and $P_{m 2}>0$ ), while in mode D (high speed cruising) MG1 operates as motor $P_{m 1}>0$ and MG2 as generator $P_{m 2}<0$ in order to make the engine operating in its optimal region. In mode $\mathrm{E}$ (deceleration)

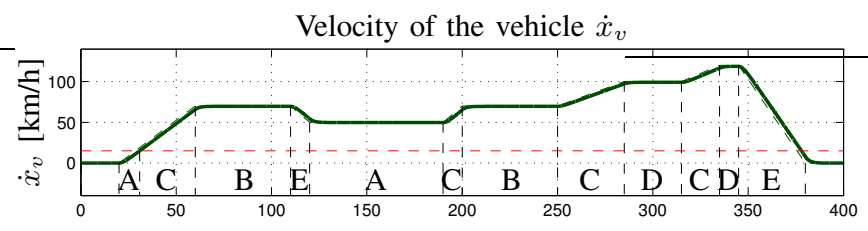

Estimated power of the vehicle $P_{v s}$

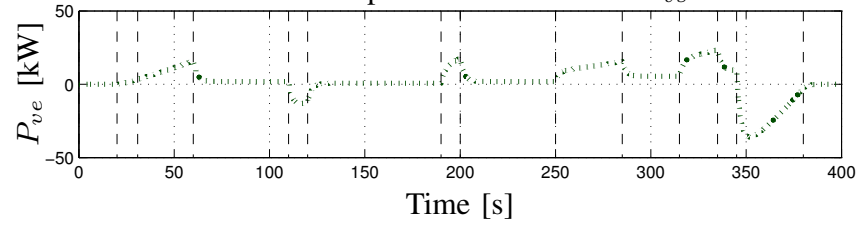

Fig. 10. Velocity of the vehicle $\dot{x}_{v}$ (green, solid) with the desired velocity $\dot{x}_{v d}$ (green, dashed) and estimated power of the vehicle $P_{v e}$ (green, dotted).
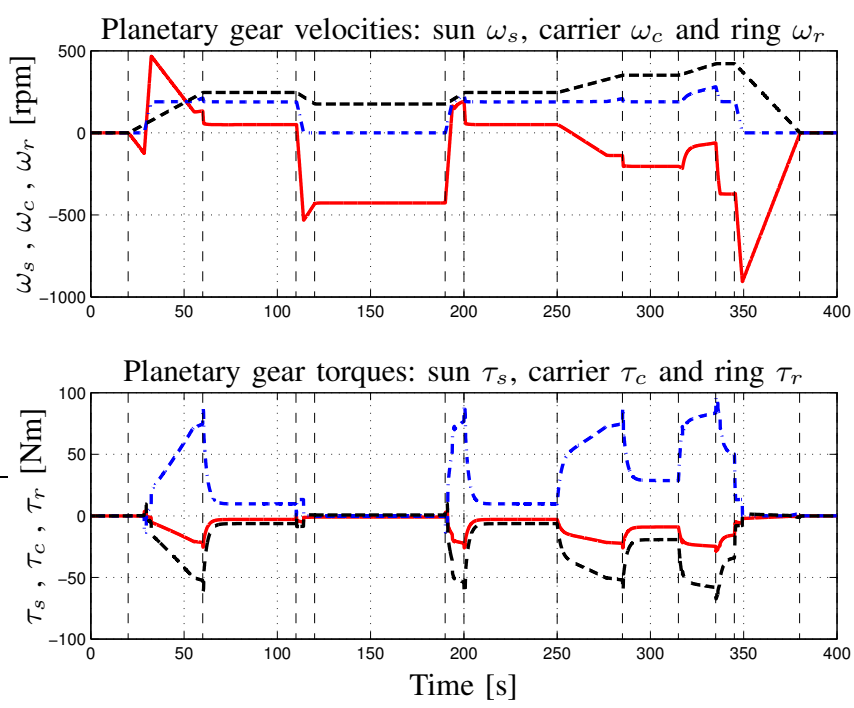

Fig. 11. Velocities and torques of the planetary gear: $\omega_{s}$ and $\tau_{s}$ (red, solid), $\omega_{c}$ and $\tau_{c}$ (blue, dash-dotted), $\omega_{r}$ and $\tau_{r}$ (black, dashed).
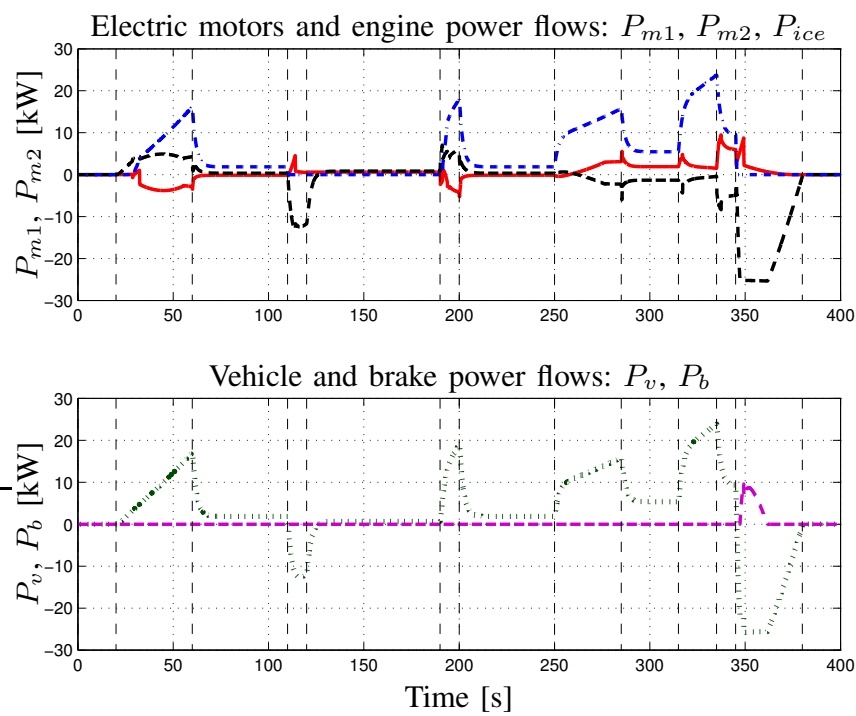

Fig. 12. Power flows into the HEV: electric motors $P_{m 1}$ (red, solid) $P_{m 2}$ (black, dashed), engine $P_{i c e}$ (blue, dash-dotted), vehicle $P_{v}$ (green, dotted), brake $P_{b}$ (pink, dashed).

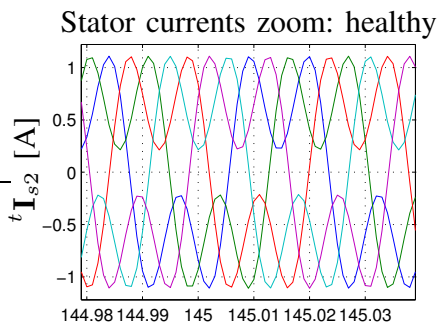

Time $[\mathrm{s}]$

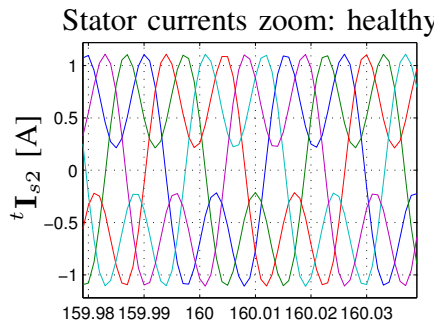

Time $[\mathrm{s}]$
Fig. 13. Zoom of stator currents of the electric motor MG2 connected to the ring.

MG2 operates as generator $P_{m 2}<0$ and the engine is shut down until a new acceleration is requested. In constant speed condition after deceleration the traction is performed by MG2 (mode A) like between 120 and 190 s. In case of strong 
deceleration a braking torque is activated, see the braking power $P_{b}$ in Fig. 12. The stator currents of MG2 are shown in Fig. 13: note that in healthy condition only the 1-st and the 3-rd harmonics are injected.

\section{B. Simulation in fault condition operation}

The simulated open-phase fault occurs at time $145 \mathrm{~s}$ and the fault-tolerant control is activated at time $160 \mathrm{~s}$. The delay time of $15 \mathrm{~s}$ has been chosen in order to show the dynamic behavior of the system in fault condition although in real application the fault detection time is less than $1 \mathrm{~s}$. The simulation results are shown from Fig. 14 to Fig. 17. When the open-phase fault occurs on the 2-nd phase of MG2, the current $I_{s 2}$ falls down to zero as shown in Fig. 17 and a torque ripple appears on the planetary gears torques, see the box in Fig. 15 and a zoom in Fig. 17. The torque ripple seems not to affect the HEV performances because the whole system behaves such a first-order filter (note that Fig. 14 does not have any noticeable differences compared to Fig. 10). However the planetary gear torque ripple could cause severe mechanical failures therefore a control reconfiguration is strongly needed. At time $160 \mathrm{~s}$ when the post-fault control is
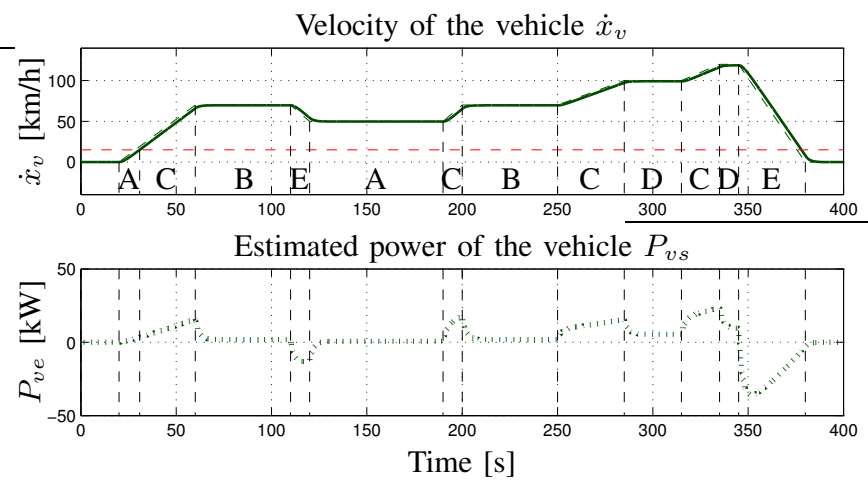

Fig. 14. Velocity of the vehicle $\dot{x}_{v}$ (green, solid) with the desired velocity $\dot{x}_{v d}$ (green, dashed) and estimated power of the vehicle $P_{v e}$ (green, dotted).
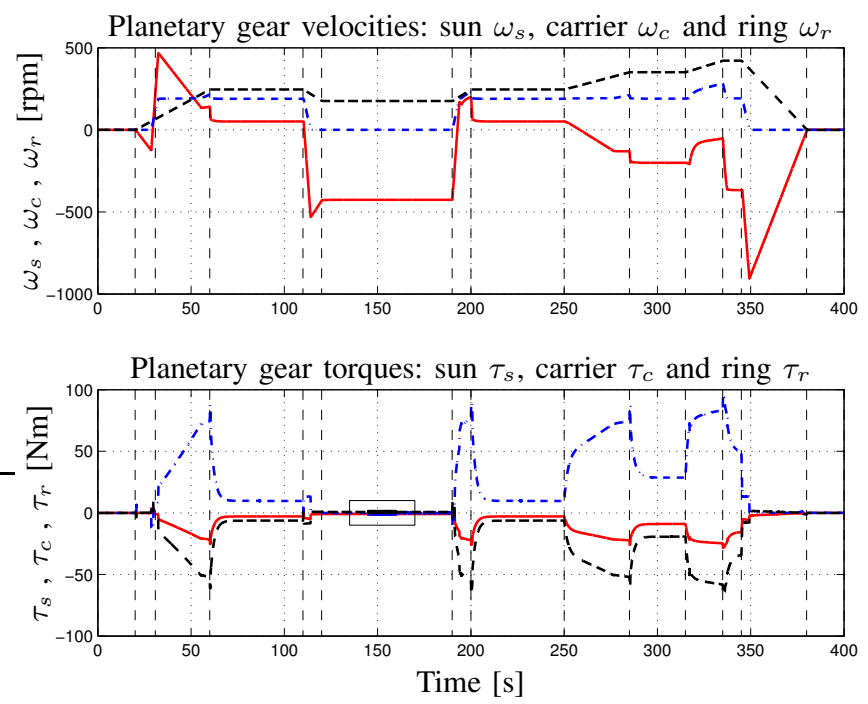

Fig. 15. Velocities and torques of the planetary gear: $\omega_{s}$ and $\tau_{s}$ (red, solid), $\omega_{c}$ and $\tau_{c}$ (blue, dash-dotted), $\omega_{r}$ and $\tau_{r}$ (black, dashed).
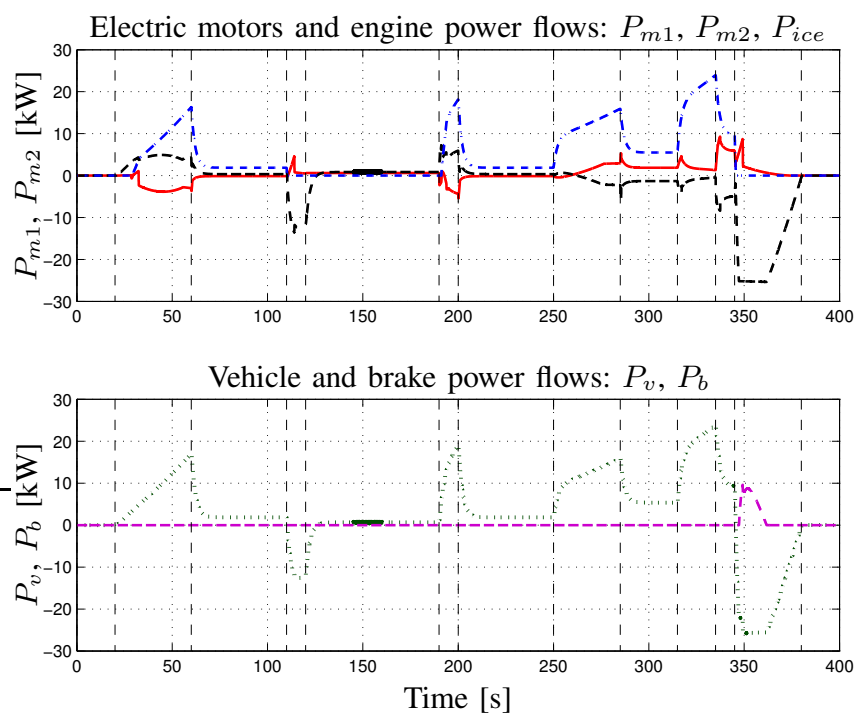

Fig. 16. Power flows into the HEV: electric motors $P_{m 1}$ (red, solid) $P_{m 2}$ (black, dashed), engine $P_{i c e}$ (blue, dash-dotted), vehicle $P_{v}$ (green, dotted), brake $P_{b}$ (pink, dashed).
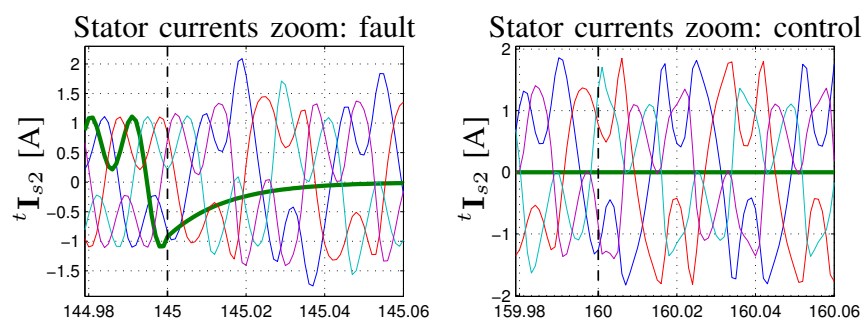

Planetary gear torques: fault
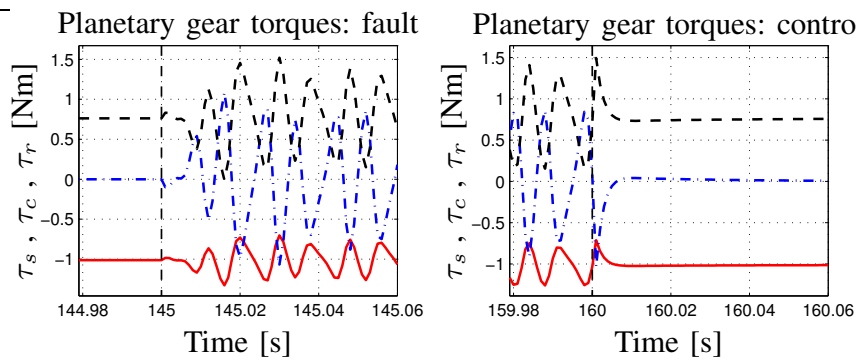

Fig. 17. Zoom of stator currents of the electric motor MG2 connected to the ring and planetary gear torques.

applied the torque ripple disappears. Since the MG2 motor drive provides the same rated power also in fault condition, then the healthy currents amplitude increases, see Fig. 17.

\section{CONCLUSIONS}

The dynamic modeling of the Toyota Hybrid System has been addressed successfully in the paper by using the POG technique which explicitly shows the power flows within the system. The case of open phase fault of a multi-phase machine has been considered occurring during the operation of the vehicle. Simulation results are provided showing the effectiveness of the model and control. The modular POG modeling approach adopted in the paper can be used to simulate also different electric and hybrid architectures. Therefore this work is the starting point for future studies on new architectures of electric and hybrid vehicles which 


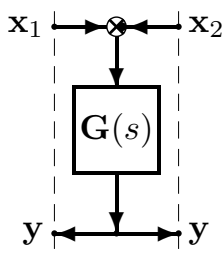

a) $e . b$.

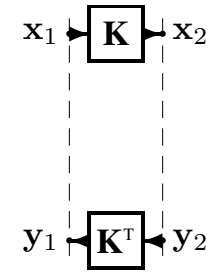

b) $c . b$.
Fig. 18. POG basic blocks: a) elaboration block; b) connection block.

exploit the advantages of multi-phase synchronous motors. Moreover new and safer control strategies can be developed and tested using the proposed simulation model which is suitable also in fault condition.

\section{APPENDIX: POWER-ORIENTED GRAPHS BASIC FEATURES}

The Power-Oriented Graphs (POG) [11]-[12] is an energybased modeling technique suitable to graphically describe the dynamic model of any type of physical system in different energetic domains: electrical, mechanical and hydraulics. The POG schemes put in evidence the power flows within the systems, they are easy to use, easy to understand even by a neophyte and can be directly implemented in Simulink. The POG block schemes are standard block diagrams combined with a modular structure based on only the two blocks shown in Fig. 18.a and Fig. 18.b: the elaboration block (e.b.) stores and/or dissipates energy (i.e. springs, masses, dampers, capacities, inductances, resistances, etc.); the connection block (c.b.) redistributes the power within the system without storing nor dissipating energy (i.e. gear reduction, transformers, etc.). The e.b. and the c.b. are can represent both scalar and vectorial systems. In the vectorial case, $\mathbf{G}(s)$ and $\mathbf{K}$ are matrices: $\mathbf{G}(s)$ is always a square matrix composed by positive real transfer functions; matrix $\mathbf{K}$ can also be rectangular. The circle present in the e.b. is a sum element and the black dot represents a minus sign that multiplies the entering variable. POG keep a direct correspondence between the dashed sections of the graphs and the real power sections of the modeled systems: the scalar product $\mathbf{x}^{\mathrm{T}} \mathbf{y}$ of the two power vectors $\mathbf{x}$ and $\mathbf{y}$ in each dashed line of a POG, see Fig. 18, has the physical meaning of power flowing through that section. Moreover the POG schemes are in direct correspondence with the state space dynamic equations.

\section{REFERENCES}

[1] C. C. Chan, "The State of the Art of Electric, Hybrid, and Fuel Cell Vehicles", Proc. of the IEEE, vol.95, no.4, pp.704-718, 2007

[2] C. C. Chan, A. Bouscayrol, and K. Chen, "Electric, Hybrid, and FuelCell Vehicles: Architectures and Modeling", IEEE Trans. On Vehicular Technology, Vol.59, no.2, pp.589-598, 2010

[3] D. W. Gao, C. Mi, A. Emadi, "Modeling and Simulation of Electric and Hybrid Vehicles", Proc. of the IEEE, vol.95, no.4, pp.729-745, 2007

[4] J. R. Bumby et al., "Computer modeling of the automotive energy requirements for internal combustion engine and battery electricpowered vehicles" Proc. Inst. Elect. Eng., vol. 132, pt. A, no. 5, pp. 265279, 1985.
[5] T. Markel, A. Brooker, T. Hendricks, V. Johnson, K. Kelly, B. Kramer, M. OKeefe, S. Sprik, K. Wipke, "ADVISOR: a systems analysis tool for advanced vehicle modeling", Journal of Power Sources, Volume 110, Issue 2, Pages 255-266, August 2002

[6] A. Rousseau, P. Sharer, F. Besnier, "Feasibility of Reusable Vehicle Modeling: Application to Hybrid Vehicles", SAE 2004-01-1618, SAE World Congress, Detroit, March 2004

[7] S. Halbach, P. Sharer, P. Pagerit, C. Folkerts, A. Rousseau, "Model Architecture, Methods, and Interfaces for Efficient Math-Based Design and Simulation of Automotive Control Systems", SAE 2010-01-0241, SAE World Congress, Detroit, April 2010

[8] Paynter, H.M., Analysis and Design of Engineering Systems, MITpress, Camb., MA, 1961

[9] D. C. Karnopp, D.L. Margolis, R. C. Rosemberg, System dynamics Modeling and Simulation of Mechatronic Systems, Wiley Interscience, ISBN 0-471-33301-8, 3rd ed. 2000.

[10] X. Guillaud, P. Degobert, J. P. Hautier, "Modeling, control and causality: the Causal Ordering Graph", 16th IMACS World Congress, CD-ROM, Lausanne (Switzerland), August 2000.

[11] R. Zanasi, "Power-Oriented Modeling of Dynamical Systems for Simulation”, MCTS'91, vol. 2, pp. 31-35, Lille, France, May 1991.

[12] R. Zanasi, "The Power-Oriented Graphs Technique: system modeling and basic properties", Proc. of IEEE Vehicular Power and Propulsion Conference (VPPC), 2010.

[13] J. C. Mercieca, J. N. Verhille, A. Bouscayrol, "Energetic Macroscopic Representation of a subway traction system for a simulation model", IEEE-ISIE'04, Ajaccio (France), May 2004

[14] A. Bouscayrol, B. Davat, B. de Fornel, B. Franois, J. P. Hautier, F. Meibody-Tabar, M. Pietrzak-David, "Multimachine Multiconverter System: application for electromechanical drives", Eur. Physics Journal - Appl. Physics, vol. 10, no. 2, pp. 131-147, May 2000.

[15] R. Zanasi, F. Grossi, "Differences and common aspects of POG and EMR energy-based graphical techniques", Proc. of IEEE Vehicle Power and Propulsion Conference (VPPC), 2011

[16] K. Muta, M. Yamazaki, and J. Tokieda, "Development of NewGeneration Hybrid System THS II-Drastic Improvement of Power Performance and Fuel Economy" SAE Technical Paper 2004-01-0064

[17] Miller, J.M., "Hybrid Electric Vehicle Propulsion System Architectures of the e-CVT Type", IEEE Trans. On Power Electronics, Vol. 21, No. 3, May 2006.

[18] Mansour, C., Clodic, D., "Dynamic modeling of the electromechanical configuration of the Toyota Hybrid System series/parallel power train", International Journal of Automotive Technology, vol.13, pp.143-166, 2012

[19] E. Levi, "Multiphase Electric Machines for Variable-Speed Applications" IEEE Trans. on Industrial Electronics, , vol.55, no.5, pp.18931909, May 2008.

[20] L. Parsa, "On advantages of multi-phase machines" 31st Annual Conference of IEEE Industrial Electronics Society, IECON 2005.

[21] R. Zanasi, F. Grossi "The POG Technique for Modeling Planetary Gears and Hybrid Automotive Systems" in Proc. of Vehicular Power and Propulsion Conference VPPC 2009

[22] R. Zanasi, F. Grossi "Modelling Hybrid Automotive Systems with the POG Technique", Journal of Asian Electric Vehicles, Volume 8, Number 1, June 2010

[23] R. Willis, "Principles of Mechanism", second edition, Longmans, Green and Co., 1870

[24] Y. Crevits, X. Kestelyn, E. Semail, "Investigation on vector control of three-phase synchronous machines under supply fault condition" Proc. of SPEEDAM 2006, pp.439-444.

[25] M. Fei, R. Zanasi, F. Grossi, "Modeling of Multi-phase Permanent Magnet Synchronous Motors under Open-phase Fault Condition", ICCA 2011 - 9th IEEE International Conference on Control \& Automation, Santiago, Chile, 2011

[26] J. Liu, H. Peng "Modeling and Control of a Power-Split Hybrid Vehicle", IEEE Trans. on Control System Technology, Vol. 16, no. 6, pp.1242-1251, November 2008

[27] K. Chen, W. Lhomme, A. Bouscayrol, A. Berthon "Comparison of two series-parallel Hybrid Electric Vehicles focusing on control structures and operation modes" Vehicular Power and Propulsion Conference VPPC 2009, Dearborn, Michigan, USA, 2009

[28] M. Fei, R. Zanasi, "Multi-phase Synchronous Motors: Minimum Dissipation Fault-Tolerant Control with Currents Saturation", Proc. of CDC 2012, 51st IEEE Conference on Decision and Control, Maui, Hawaii, USA 2012. 